\title{
The role of water channel proteins and nitric oxide signaling in rice seed germination
}

\author{
Hong-Yan Liu ${ }^{1}$, Xin $\mathrm{Yu}^{1}$, Da-Yong Cui ${ }^{1}$, Mei-Hao Sun ${ }^{1}$, Wei-Ning Sun ${ }^{1}$, Zhang-Cheng Tang ${ }^{1}$, Sang-Soo Kwak ${ }^{2}$, \\ Wei-Ai Su${ }^{1}$ \\ ${ }^{1}$ Institute of Plant Physiology and Ecology, Shanghai Institutes for Biological Sciences, Chinese Academy of Sciences, 300 Fenglin \\ Road, Shanghai 200032, China, ${ }^{2}$ Korea Research Institute of Bioscience and Biotechnology, Oun-dong 52, Yusong, Taejon 305-333, \\ South Korea
}

Previous studies have demonstrated the possible role of several aquaporins in seed germination. But systematic investigation of the role of aquaporin family members in this process is lacking. Here, the developmental regulation of plasma membrane intrinsic protein (PIP) expression throughout germination and post-germination processes in rice embryos was analyzed. The expression patterns of the PIPs suggest these aquaporins play different roles in seed germination and seedling growth. Partial silencing of the water channel genes, OSPIP1;1 and OSPIP1;3, reduced seed germination while over-expression of $O_{S} P I P 1 ; 3$ promoted seed germination under water-stress conditions. Moreover, spatial expression analysis indicates that $O S P I P 1 ; 3$ is expressed predominantly in embryo during seed germination. Our data also revealed that the nitric oxide (NO) donors, sodium nitroprusside (SNP) and $S$-nitrosoglutathione (GSNO), promoted seed germination; furthermore, the NO scavenger, 2-(4-carboxyphenyl)-4,4,5,5-tetramethylimidazoline-1-oxyl-3-oxide, inhibited germination and reduced the stimulative effects of SNP and GSNO on rice germination. Exogenous NO stimulated the transcription of $O_{S P I P 1 ; 1, O S P I P 1 ; 2, O S P I P 1 ; 3}$ and $O S P I P 2 ; 8$ in germinating seeds. These results suggest that water channels play an important role in seed germination, acting, at least partly, in response to the NO signaling pathway.

Keywords: germination, nitric oxide, Oryza sativa, over-expression, silencing, water channel

Cell Research (2007) 17:638-649. doi: 10.1038/cr.2007.34; publication online 24 April 2007

\section{Introduction}

Seed germination begins with uptake of water by the dry seed and terminates with radicle emergence and elongation of the embryonic axis [1]. Water movement into living cells governs seed germination. Previous studies have shown that aquaporins control intra- and intercellular water flow in plant cells [2]. There are 33 aquaporin genes in rice, of which 11 are plasma membrane intrinsic protein genes (PIPs) [3, 4], and they can be divided into two groups, PIP1 and PIP2 as that in Arabidopsis thaliana [5] and Zea mays

Correspondence: Wei-Ai Su

Tel: +86-21-54924247; Fax: +86-21-54924015

E-mail: zstressc@online.sh.cn

Received 31 October 2006; revised 10 January 2007; accepted 25 January 2007; publish online 24 April 2007
[6]. Plant PIP2 proteins have high water channel activity in Xenopus oocytes, whereas PIP1 proteins are often inactive or have low activity [7]. Some PIP1 members may not be related to water movement across the membrane but they might act as transporters for small solutes or gases. To investigate genes involved in the control of Medicago truncatula germination, suppressive subtractive hybridization was used to clone several genes including AQP1, a $\gamma$-TIP member [8]. Gao et al. [9] have demonstrated that BnPIPI and the tonoplast intrinsic protein, BnTIP2, are involved in seed germination. In the present work, we investigated the role of PIPs in rice seed germination to further reveal the functions of the water channel family.

Increasing experimental data have demonstrated that the germination of seeds is controlled by a variety of positive and negative regulatory signals: GA, ABA, light, chilling, sugars and nitric oxide (NO) $[10,11]$. NO is a gaseous free radical that diffuses readily through membranes. Previous 
studies have shown the presence of NO in plants and its functions in many biological processes, including seed germination, leaf extension, root development, respiration, plant defense and hypersensitive cell death [12-14]. However, NO exerts both beneficial and harmful effects on plants, depending on its concentrations in cells [15]. Several papers have reported that NO is an endogenous regulator of seed dormancy, illustrated by the NO donor compounds breaking dormancy of Arabidopsis seeds [1618] and reducing dormancy of three barley cultivars [17]. Conversely, NO scavengers have been shown to strengthen dormancy of Arabidopsis and barley seeds [17, 18], and reduce the stimulative effects of NO donors on seed germination [10, 17]. Beligni and Lamattina [10] concluded that NO was involved in light-mediated signaling, such as light stimulation of seed germination, de-etiolation and inhibition of hypocotyl elongation. Kopyra and Gwóźdź [19] described that NO was a potent germination stimulator for the faster germination rate of lupin seeds. In addition, the endogenous NO concentration peak in homogenates from sorghum embryonic axes was reached at 24 to $30 \mathrm{~h}$ after imbibition, which is in coincidence with the initiation of germination [20].

Here, we report the expression, regulation and gene manipulation of PIPs during rice seed germination and early seedling growth. According to our data, we conclude that water channel proteins play an important role in the progress of seed germination, probably in part in response to the NO signaling pathway.

\section{Materials and Methods}

\section{Plant materials}

Rice (Oryza sativa L. Japonica cv. Zhonghua 11) was used for transformation of SWPA2-OSPIP1;3 sense and antisense construct. The $O s P I P 1 ; 3$ gene and its upstream sequence was amplified from rice genomic DNA with forward primer 5'-TTG CATCTAGAG CTC GGT TGA TTA TTT C-3' and reverse primer 5'-CAA GAG CTCTAGAAT GGA GGG GAA GGA G-3'. Restriction enzyme sites are underlined. The PCR fragment was digested with $X b a \mathrm{I}$ and inserted into the binary vector pCAMBIA 1301 between the SWPA2 promoter and a nopaline synthetase terminator in a reverse orientation. Agrobacterium tumefaciens strain EHA105 was used to transform this resulting construct. The stress-inducible SWPA2 promoter was strongly induced in response to environmental stresses [21, 22]. Transformation and regeneration of transgenic rice was performed according to the protocol as described previously [23].

\section{Seed germination}

The O. sativa ssp Japonica cv. Zhonghua11 wild type, SWPA2OsPIP 1;3 sense and antisense seeds $\left(\mathrm{T}_{2}\right)$ were derived from parent plants that were grown at the same time and next to each other in the same green house. Seeds were harvested and dried before being kept in sealed jars. In each experiment, at least three Petri dishes of seeds were imbibed at $4{ }^{\circ} \mathrm{C}$ for $3 \mathrm{~d}$ before being transferred to $28^{\circ} \mathrm{C}$ for germination. Seeds were judged to be germinated when their radicles pierced the seed coat. For the seeds that were germinated in PEG, 15\% PEG6000 was used to replace water. The imbibition and germination conditions remain the same.

To investigate the effect of $\mathrm{NO}$ on seed germination, the NO donors sodium nitroprusside (SNP) (100 $\mu \mathrm{M}$, Invitrogen), S-nitrosoglutathione (GSNO) $(100 \mu \mathrm{M}$, Sigma), the NO scavenger PTIO (2(4-carboxyphenyl)-4,4,5,5-tetramethylimidazoline-1-oxyl-3-oxide) $(100 \mu \mathrm{M}$, Invitrogen) and in combination with SNP or GSNO were used to imbibe Nipponbare seeds at $28^{\circ} \mathrm{C}$. To check the effect of cyanide on seed germination, potassium ferricyanide (100 $\mu \mathrm{M}$, Sigma) was also used to imbibe Nipponbare seeds at $28^{\circ} \mathrm{C}$. The Petri dishes were wrapped with parafilm during the experiment.

\section{RNA preparation}

RNA was extracted from embryos or seedlings using Trizol RNA isolation reagent (Invitrogen, Carlsbad, CA, USA) according to the manufacturer's instructions. Embryos were isolated from dry seeds (kept in $28^{\circ} \mathrm{C}$ for $3 \mathrm{~d}$ ), seeds imbibed for $3 \mathrm{~d}$ at $4{ }^{\circ} \mathrm{C}(0 \mathrm{~h})$ and germinating seeds (incubated at $28^{\circ} \mathrm{C}$ for $12,24,30,36,48$ and $72 \mathrm{~h}$ ). To determine the expression level of OSPIP1;3 in antisense plants, total RNA was also extracted from 2-week-old $\mathrm{T}_{2}$ plants and wild-type plants. To determine PIP1 transcripts in wild type, sense-transgenic and silenced-transgenic seeds, embryos were isolated from the seeds germinated for $36 \mathrm{~h}$ in the presence or absence of $15 \%$ PEG. To prevent RNA contamination from genomic DNA, total RNA was treated with RNase-free DNase II (Invitrogen, Carlsbad, CA, USA) according to the manufacturer's instructions.

\section{Real-time PCR}

Real-time PCR quantification of RNA targets was performed in the Rotor-Gene 3000 real-time thermal cycling system (Corbett). A rice polyubiquitin gene $(U B Q)$ [24] was used as a control in the real-time PCR reactions. A two-step RT-PCR procedure was performed in all experiments. First, total RNA samples $(2 \mu \mathrm{g}$ per reaction) were reversely transcribed into cDNAs by AMV reverse transcriptase according to the manufacturer' s instructions (TaKaRa, Japan). Then the cDNAs were used as templates in real-time PCR reactions with gene-specific primers and probes (designed and synthesized by GeneCore, Shanghai; Table 1). The amplification reactions were performed in a total volume of $25 \mu$. The reaction mixture contained $2 \mu \mathrm{l}$ of cDNA, $0.5 \mu \mathrm{l}$ of the forward and reverse primers $(10 \mu \mathrm{M})$ along with $0.5 \mu \mathrm{l}$ of the TaqMan-MGB probe $(5 \mu \mathrm{M})$ corresponding to each primer and probe, $0.25 \mu \mathrm{l}$ of enzymes (TaKaRa), $0.75 \mu$ of dNTP $(10 \mathrm{mM}) ; 0.5 \mu l$ of $\mathrm{MgCl}_{2}(250 \mathrm{mM})$; $5 \mu 1$ of $5 \times$ real-time PCR buffer and $15 \mu$ of PCR-grade sterile water. The Rotor-Gene 3000 cycler was programmed as follows: $4 \mathrm{~min}$ at $94{ }^{\circ} \mathrm{C} ; 60$ cycles of $30 \mathrm{~s}$ at $94^{\circ} \mathrm{C}$ and $30 \mathrm{~s}$ at $60^{\circ} \mathrm{C}$. A control without the corresponding template DNA was included in every quantitative PCR assay for each primer and probe set. The fluorescence signal was carried out during the annealing step. All experiments were repeated three times. The real-time amplification data were analyzed using Rotor-Gene 3000 software (Corbett). The relative expression was evaluated using the comparative cycle threshold method taking UBQ as the reference gene.

\section{$P C R$}

PCR strategy was used to identify the silenced-transgenic plant. To avoid the interference of endogenous OsPIP1;3, PCR was car- 
Table 1 Gene-specific primers and TaqMan-MGB probes used in the real-time PCR experiments

\begin{tabular}{|c|c|c|}
\hline Name & cDNA GenBank Accession No. & Probe, forward and reverse primers \\
\hline \multirow[t]{2}{*}{ OsPIP $1 ; 1$} & AJ224327 & FAM (5'-3') TGTAGTTGCCAGTGGCT \\
\hline & & FP (5'-3') TACATGGGCAATGGCGGT \\
\hline \multirow[t]{2}{*}{ OsPIP $1 ; 2$} & AK098849 & FAM (5'-3') ACCGTCCTCACCGTCAT \\
\hline & & FP (5'-3') GGCCACCTTCCТCTTCCTCTAC \\
\hline \multirow{2}{*}{ OsPIP $1 ; 3$} & & FP (5'-3') AAGGACTACCGGGAGCCG \\
\hline & & RP (5'-3') GGTAGAACGACCACGACGTCA \\
\hline \multirow[t]{2}{*}{ OsPIP $2 ; 1$} & AK 072519 & FAM (5'-3') TCGGCCTCGTCAAGG \\
\hline & & FP (5'-3') AGTGCCTCGGCGCCAT \\
\hline OsPIP $2 ; 2$ & & RP (5'-3') GGCACTGCGCGATGATGTA \\
\hline \multirow[t]{3}{*}{ OsPIP $2 ; 3$} & AL662958 (DNA No.) & FAM (5'-3') AAGAGGAGCAAGCACGG \\
\hline & & FP (5'-3') TCTTCTCCGCCACCGACC \\
\hline & & RP (5'-3') AACACCGCGAACCCAATTG \\
\hline \multirow[t]{3}{*}{ OsPIP $2 ; 4$} & AK072632 & FAM (5'-3') ACTCACATGTTCCCGTGTT \\
\hline & & FP (5'-3') ACCGATCCCAAGCGCAA \\
\hline & & RP (5'-3') GCGAACCCGATTGGCAG \\
\hline \multirow[t]{2}{*}{ OsPIP $2 ; 5$} & AK107700 & FAM (5'-3') CGACCATCCCCGTCAC \\
\hline & & FP (5'-3') CCGTGTTCATGGTGCACCT \\
\hline OsPIP $2 ; 7$ & & RP (5'-3') ATGAGCGTCGCCATGAACTC \\
\hline \multirow[t]{3}{*}{ OsPIP $2 ; 8$} & AK109024 & FAM (5'-3') CTGCTGTTGGTGTGCAT \\
\hline & & FP (5'-3') CATTGCGGAGTTCACAGCC \\
\hline & & RP (5'-3') CCGATCACGGTGCTCACA \\
\hline \multirow[t]{3}{*}{$U B Q$} & D12776 & FAM (5'-3') CACCACAGCACACGC \\
\hline & & FP (5'-3') TTGTCCTGCGCCTCCGT \\
\hline & & RP (5'-3') GCATAGGTATAATGAAGTCCAATGC \\
\hline
\end{tabular}

ried out with the primers 5'-TTG CCA AAT CCT ATC ATA CAC-3' and 5'-CTG GGT TGG TCC GTT CAT-3', which were based on the sequence of promoter $S W P A 2$ and $O S P I P 1 ; 3$, respectively, at $94{ }^{\circ} \mathrm{C}$ for $4 \mathrm{~min}$, followed by 30 cycles at $94^{\circ} \mathrm{C}$ for $45 \mathrm{~s}, 56^{\circ} \mathrm{C}$ for $1 \mathrm{~min}$ and $72{ }^{\circ} \mathrm{C}$ for $1 \mathrm{~min}$, and finished by an extension at $72{ }^{\circ} \mathrm{C}$ for $10 \mathrm{~min}$.

Plasma membrane protein extraction and western blot

Embryos were isolated from dry seeds and germinating seeds (12 and $24 \mathrm{~h}$ after germination). Embryo plasma membrane fractions were prepared by the aqueous two-phase partitioning method as described previously [25]. Membrane protein concentration was measured according to Bradford [26]. Western blots were performed essentially by the standard method described by Sambrook et al. [27]. Proteins $(20 \mu \mathrm{g})$ were separated by SDS-PAGE (12\% acrylamide), transferred to nitrocellulose membranes and blocked with $5 \%$ skim milk in phosphate-buffered saline overnight. Rabbit serum raised against the N-terminal conserved peptide, KDYNEPPPAPLFEPGELSSWS, of PIP1 conjugated to bovine serum albumin was used as the primary 
antibody (1:500) and anti-rabbit IgG (1:1000) conjugated to alkaline phosphatase as the secondary antibody. The signals were detected by the 5-bromo-4-chloro-3-indolyphsophate/nitro-blue tetrazolium (NBT/BICP) method.

\section{Histochemical staining of GUS activity}

To localize the OSPIP1;3 expression in seeds during germination, rice seeds harboring the $O S P I P 1 ; 3$ promoter::GUS fusions gene [28] were germinated for $24 \mathrm{~h}$ in water, split in half and stained for $2 \mathrm{~h}$ at $37^{\circ} \mathrm{C}$ in GUS assay buffer $(10 \mathrm{mM}$ phosphate buffer $(\mathrm{pH} 7.0)$, $50 \mathrm{mM}$ EDTA, $0.5 \mathrm{mM}$ potassium ferrocyanide, $0.5 \mathrm{mM}$ potassium ferricyanide, $0.1 \%$ Triton X-100, $1 \mathrm{mg} / \mathrm{ml}$ 5-bromo-4-chloro-3-indolyl- $\beta$-D-glucupyranoside (X-gluc)), destained with 70\% ethanol and then photographed. To further detect GUS activities in embryos, the embryos detached from the seed samples were transferred into FAA solution ( $5 \%$ formaldehyde, $5 \%$ acetic acid, $63 \%$ ethanol and $27 \% \mathrm{H}_{2} \mathrm{O}$ ). Subsequently, the materials were vacuum-infiltrated for $30 \mathrm{~min}$ and then incubated at $4{ }^{\circ} \mathrm{C}$ overnight. The samples in FAA solution were dehydrated with graded ethanol series and then embedded into Epon812-resin. The samples were polymerized at $60^{\circ} \mathrm{C}$ and cut into 5 - $\mu \mathrm{m}$-thick sections. Sections were microscopically observed (Leica DMR, Germany) and photographed.

\section{Water uptake analysis}

The amount of absorbed water by the embryos, which were separated from wild type and silenced-transgenic seeds, was determined as the increase in weight of imbibed embryos with respect to the initial dry weight of the embryos. Prior to weighing, embryos were paper-blotted for $10 \mathrm{~s}$.

\section{NO assay}

Seed embryos ( $0.5 \mathrm{~g}$ fresh weight $)$ were homogenized in $5 \mathrm{ml}$ of extraction buffer ( $40 \mathrm{mM}$ HEPES, $\mathrm{pH}$ 7.2). The homogenate was filtered with gauze and then centrifuged $(5000 \times \mathrm{g}, 10 \mathrm{~min})$ at $4{ }^{\circ} \mathrm{C}$. The supernatants were used for NO determination using the Griess reagent system (Promega).

\section{Results}

Expression patterns of PIPs during rice seed germination and post-germination

Real-time PCR was performed to analyze the accumulation patterns of each of 11 PIPs in dry embryos and during the germination and post-germination phases at intervals from 0 to $72 \mathrm{~h}$ after germination (Figure 1A). Except for OsPIP1;3, OsPIP2;4 and OsPIP2;5, other OsPIPs were expressed in dry seeds at different expression levels. The expression of OsPIP1;1, OsPIP1;2, OsPIP2;7 and Os$P I P 2 ; 8$ markedly decreased after imbibition at $4{ }^{\circ} \mathrm{C}$ for $3 \mathrm{~d}$. After germination, expression of most $O s P I P$ s started to increase within $24 \mathrm{~h}$. Among these, $O S P I P 1 ; 1$ and $O s-$ $P I P 1 ; 2$ expression increased within $12 \mathrm{~h}$, but OsPIP2;2, OsPIP 2;3 and OsPIP2;6 were not induced markedly until $30 \mathrm{~h}$ after germination. Unlike most PIPs, whose expression peaked before radicle emergence and declined immediately afterwards, the levels of OSPIP 2;2 and OSPIP 2;6 increased during germination and were maintained during the post-germination phase. The distinct PIP expression pattern suggests that specific isoforms of PIPs could play different roles in seed germination.

In addition, western blot was used to analyze the changes of PIP1 protein abundance in embryos of seeds during germination. Figure 1B shows the results of a western blot analysis using purified antibody raised against a conserved $\mathrm{N}$-terminal peptide of PIPs (Materials and Methods). According to the comparison of the sequences, the antibody should react mainly with OsPIP1;1 and OsPIP1;2. PIP1 protein abundance was stronger in the embryos after $24 \mathrm{~h}$ of germination than that in the embryos after $12 \mathrm{~h}$ of germination. In addition, PIP1 proteins were expressed in dry embryos at very low levels (Figure 1B), indicating that the time of PIP1 protein expression is later than that of PIP1 mRNA expression. However, PIP1 protein abundance increased during seed germination and exhibited a regulation pattern essentially similar to that of the transcript expression level of PIP1 genes.

\section{Spatial expression of the OSPIP1;3 promoter-driven GUS}

The GUS activity was determined histochemically to analyze the pattern of GUS expression and infer the promoter activity of the endogenous water channel gene. The OSPIP $1 ; 3: \because G U S$ construct, harboring a $1.2 \mathrm{~kb}$ promoter, was transcribed in all the organs such as the root, stem, leaf, flower, immature embryo and the immature seed coat [28]. The GUS activity was distinctly high in the embryos of the 24-h-imbibed transgenic rice seeds in comparison to the seed coat and the endosperm (Figure 2A). In addition, a high level of GUS expression was also observed in the radicle (Figure 2B). The distribution pattern of GUS expression suggests that OsPIP1;3 could be involved in the events of seed germination.

Characterization of plants and seeds with altered expression of OsPIP1;3

We previously described the role of $O S P I P 1 ; 3$ overexpression (also known as RWC3) in drought avoidance in transgenic lowland rice [22]. To further explore water channel functions during seed germination, the expression of OsPIP1;3 was suppressed using an antisense-silencing construct. The $O S P I P 1 ; 3$ gene was cloned in an antisense orientation behind the SWPA2 promoter [21] and transformed into wild-type rice. Eleven independent transgenic lines were selected based on their hygromycin-resistant phenotype. PCR was used to confirm the identity of transgenic plants. In order to avoid PCR detection of endogenous OSPIP 1;3, we designed the forward primer and the reverse primer based on the sequence of the SWPA2 promoter and 

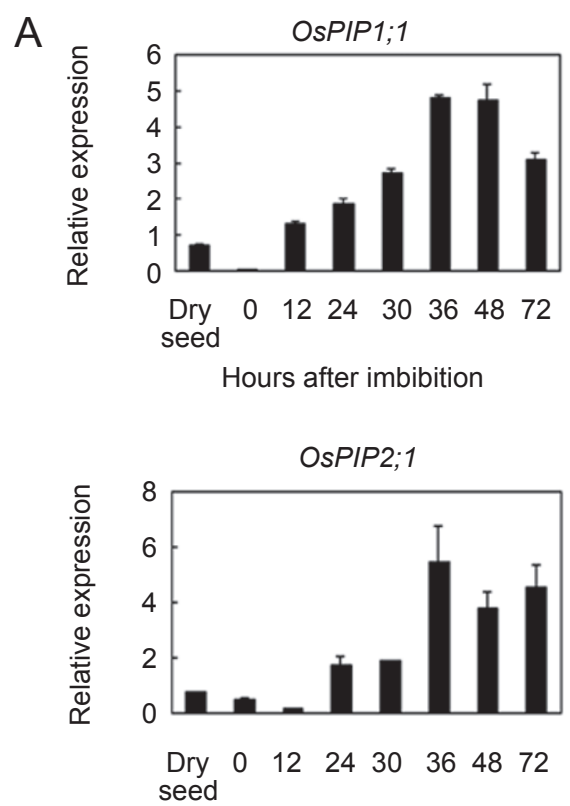

Hours after imbibition
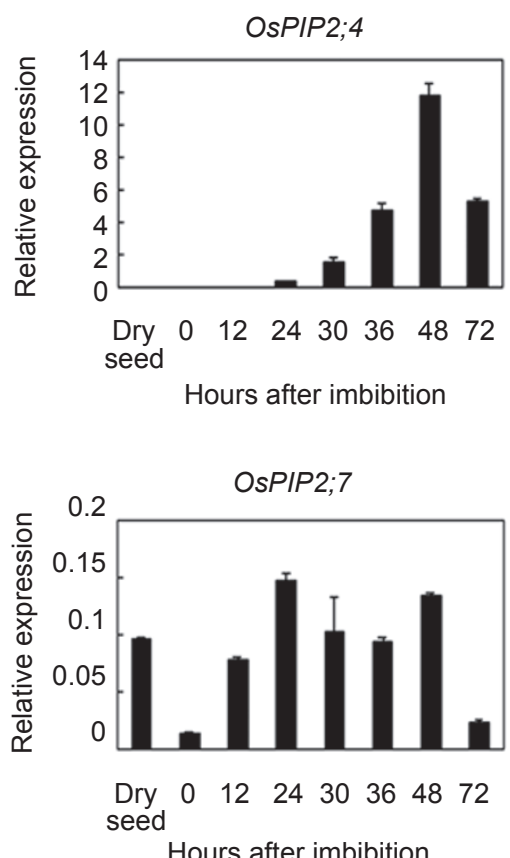
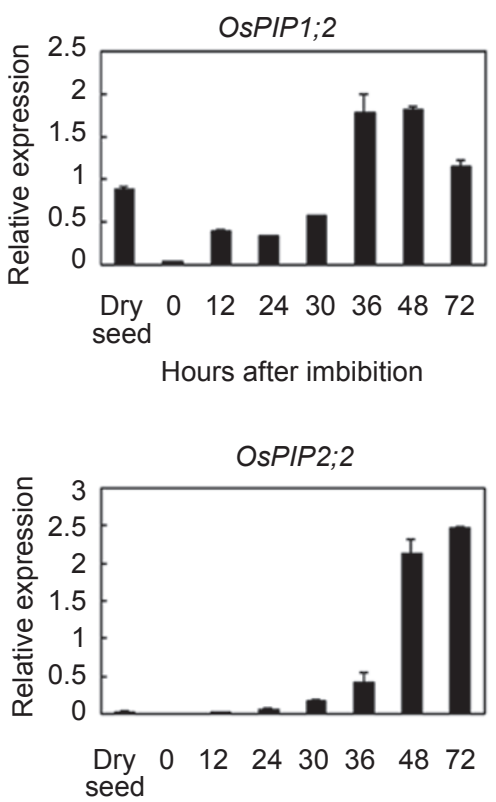

Hours after imbibition
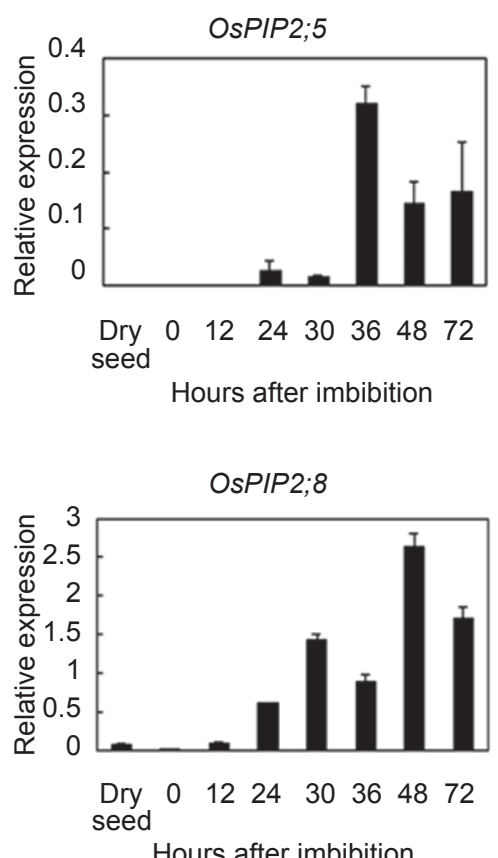

Hours after imbibition

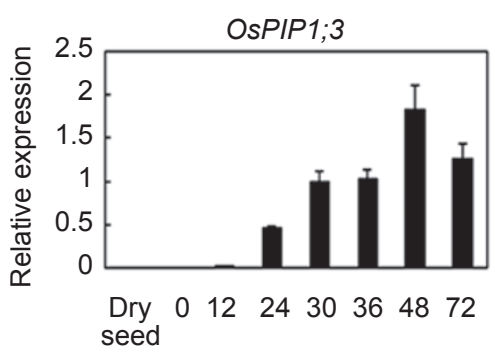

Hours after imbibition

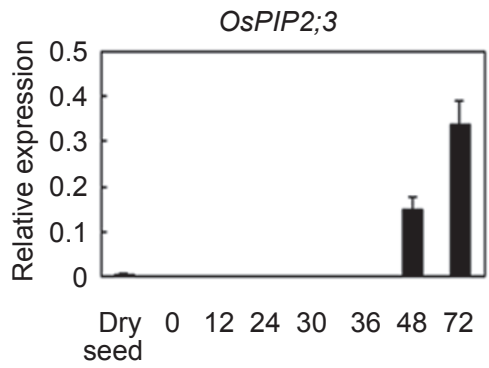

Hours after imbibition

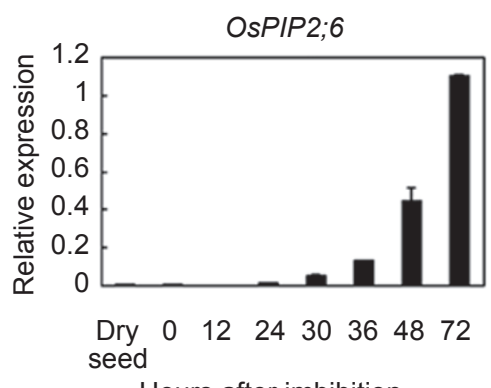

B

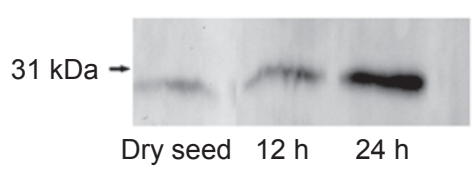

Figure 1 PIP expression patterns in the rice embryos at various times throughout germination and post-germination. (A) $P I P$ expression was determined by real-time PCR. Expression levels of PIP genes were normalized to levels of $U B Q$. Each data point represents the mean \pm S.E.M. of three independent experiments. (B) Western blot analysis of the PIP1 protein levels in the embryos of rice seeds. The rice seeds were imbibed in water for $12 \mathrm{~h}$ and $24 \mathrm{~h}$. Twenty micrograms of plasma membrane protein was loaded.

OsPIP $1 ; 3$, respectively. As representative examples, Figure $3 \mathrm{~A}$ shows that $700 \mathrm{bp}$ PCR products were amplified specifically from genomic DNA in three lines of the $\mathrm{T}_{2}$ transgenic plants. Real-time PCR analysis revealed that three different $\mathrm{T}_{2}$ OsPIP 1; 3 antisense lines expressed $O S P I P 1 ; 3$ at reduced levels, which range from $27 \%$ to $60 \%$ of the wild-type 

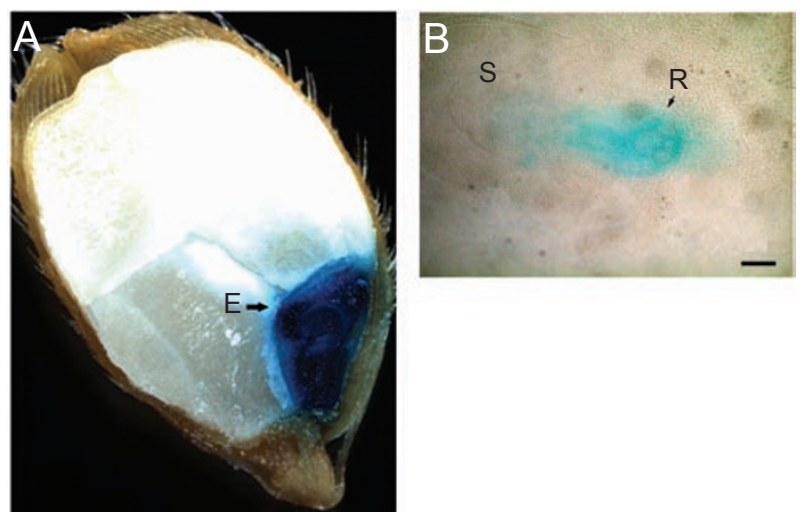

Figure 2 Tissue-specific expression analysis of the GUS reporter controlled by the OSPIP1;3 gene promoter. (A) Localization of OSPIP 1;3::GUS expression in the embryos of 24-h-imbibed seeds is shown. Seeds were split in half and then stained with GUS solution. (B) Strong GUS expression in the radicles of embryos is shown. The embryos were cut into 5 - $\mu \mathrm{m}$-thick sections. Sections were microscopically observed (Leica DMR, Germany) and photographed. E: embryo; R: radicle; S: shoot. Scale bar $=150 \mu \mathrm{m}$. level at normal growth conditions (Figure 3B). Although $S W A P 2$ promoter is a stress-inducible promoter, it has a basal expression about one-fourth to the $35 \mathrm{~S}$ promoter at normal growth conditions [21]. Therefore, the antisense plants have reduced expression levels of OsPIP $1 ; 3$ even at non-inducible conditions. The OsPIP1,3 antisense lines A27, A46 and A68 were used in further experiments. We traced the expression pattern of OSPIP1;3 in wild type, sense-transgenic and antisense-transgenic seeds treated with distilled water or water deficit mediated by PEG 6000 for $36 \mathrm{~h}$. The sense-transgenic seeds had approximately the equivalent amount of OsPIP 1;3 mRNA under normal conditions, while they had an obviously increased OsPIP 1;3 expression compared with the wild-type seeds under the water-deficit condition (Figure $3 \mathrm{C}$ ). The mRNA level of OSPIP 1;3 decreased in the antisense-transgenic seeds both under normal conditions and upon water deficit compared with the wild-type seeds, and it was lower under water-deficit conditions (Figure 3C). These results suggest
A

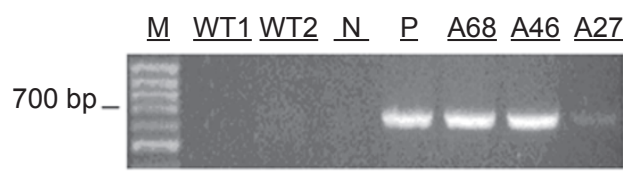

B

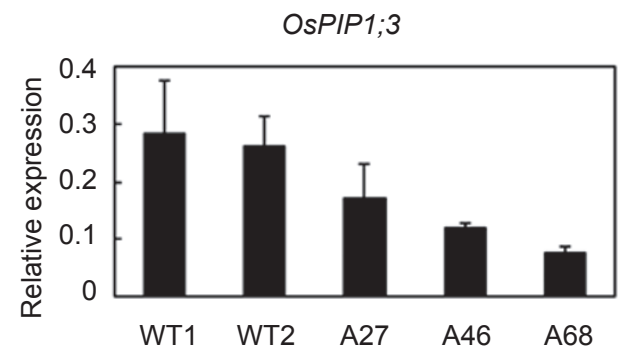

C
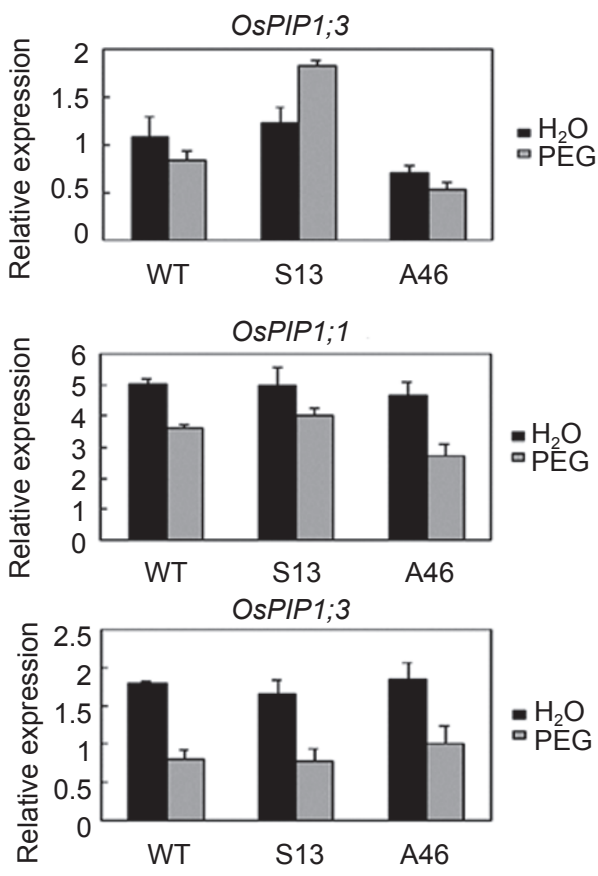

Figure 3 Characterization of $O s P I P 1 ; 3$ antisense-transgenic rice lines, sense-transgenic and antisense-transgenic seeds. (A) PCR analysis of the genomic DNA in transgenic plants. PCR was carried out with the primers 5'-TTG CCA AAT CCT ATC ATA CAC3' and 5'-CTG GGT TGG TCC GTT CAT-3'. PCR products amplified from the genomic DNA of wild type (WT1 and WT2) and three lines of antisense-transgenic plants (A27, A46 and A68) are shown. Other lanes represent: plasmid PCAMBIA 1301-SWPA2:: OsPIP1;3 (P); DNA marker (M); water as template (N). (B) Real-time PCR showed that antisense-transgenic plants exhibited reduced expression of $O S P I P 1 ; 3$. The intensity of $O S P I P 1 ; 3$ signal was normalized against $U B Q$ signal. Data are the means \pm S.E.M. of three independent experiments. (C) Real-time PCR showed the expression of OsPIP1;3, OsPIP1;1 and OsPIP1;2 in wild type (WT), sense-transgenic (S13) and antisense-transgenic seeds (A46) imbibed in water or under water deficit induced by $15 \%$ PEG6000 solutions. The intensity of these three PIP signals was normalized against $U B Q$ signal. Data are the means \pm S.E.M. of three independent experiments. 
that the stress-inducible $S W P A 2$ promoter could regulate the expression of OsPIP 1;3 gene in the seeds of transgenic rice under drought stress.

The amino-acid sequences are highly conserved among three members of the OsPIP1 group with identities of more than $80 \%$; therefore, we also examined the expression of OsPIP 1;1 and OsPIP 1;2 in the transgenic seeds. As shown in Figure 3C, both at normal growth condition and upon water deficit, the mRNA level of OSPIP 1;1 had no difference between sense-transgenic seeds and the wild type seeds; however, it was reduced in the antisense-transgenic seeds upon water deficit. The expression of OsPIP1;2 transcript was unaffected both in sense-transgenic and in antisensetransgenic seeds (Figure 3C).

\section{Effects of OsPIPs on seed germination}

Figure 1A shows that $O S P I P 1 ; 3$ expression was weak at $12 \mathrm{~h}$ after germination and started to increase at $24 \mathrm{~h}$, peaked at $48 \mathrm{~h}$, then declined at $72 \mathrm{~h}$. The developmental regulation of OSPIP 1;3 in wild-type seeds is consistent with the progress of seed germination, which started at $24 \mathrm{~h}$ and terminated at $48 \mathrm{~h}$ (Figure 4A). We analyzed the germination of OsPIP1;3 sense, antisense and wild-type rice seeds systematically. After $48 \mathrm{~h}$ of germination, $90 \%$ of wild- type seeds and $92 \%$ of sense-transgenic seeds germinated, but only $60 \%$ of antisense-transgenic seeds germinated (Figure 4A and 4B). Although $70 \%$ of the antisense seeds eventually germinated, this occurred as late as the fourth day after germination (Figure 4A). These results suggest that $O S P I P 1 ; 3$ is required for seed germination in rice. In addition, OsPIP 1; 1 may be another required component for rice seed germination, as its expression was also partially reduced in the $O S P I P 1 ; 3$ antisense-transgenic seeds.

As shown in Figure 4C and 4D, wild type and antisensetransgenic seeds exhibited significantly reduced germination rate when germinated in $15 \%$ PEG, while seeds with over-expressed $O S P I P 1 ; 3$ germinated normally under the same conditions. In addition, the final germination percentage from sense lines was higher than that of wild type (Figure 4C). The germination rate of the antisense lines was substantially inhibited under water-stress conditions with only $8 \%$ germination at $30 \mathrm{~h}$ after germination (Figure 4C). These observations suggested that increased water channel levels might provide seeds with additional ability to withstand water deficit. Taken together, these data further indicate that water channels play an important role in seed germination.

Because the rate of germination was significantly
A

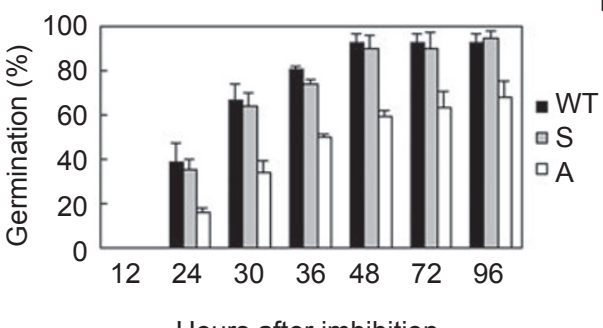

Hours after imbibition

C

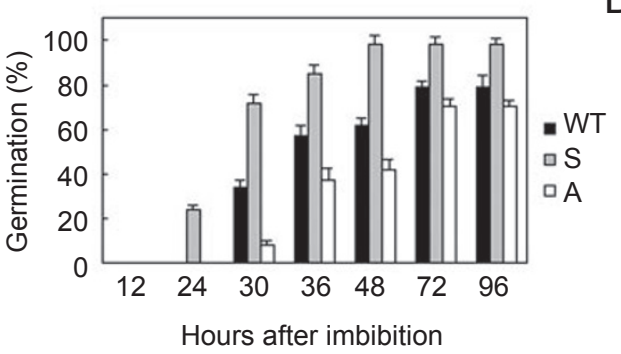

D
B

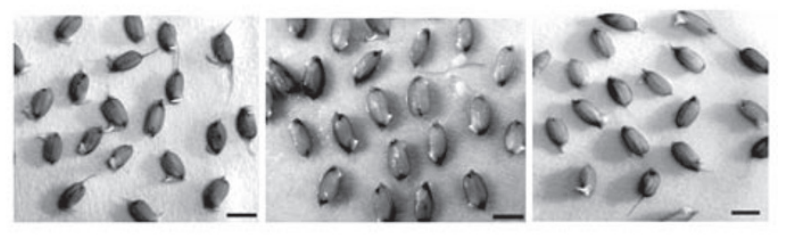

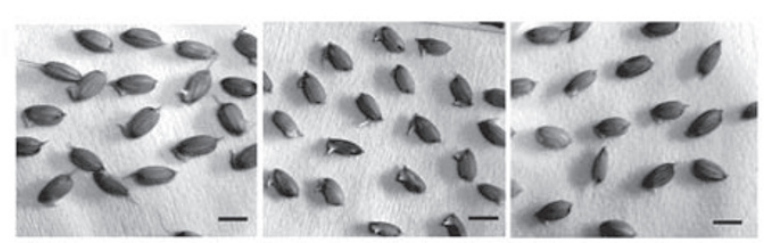

Figure 4 Phenotypic characterization of seeds from sense (S) and antisense (A) plants. (A) Time course of germination of WT, sensetransgenic and antisense-transgenic seeds in water. The percentage of germinated seeds corresponds to the mean of three experiments of 50 seeds. (B) Seeds of WT (left), sense (middle) and antisense-transgenic (right) lines corresponding to the $48 \mathrm{~h}$ time point in (A). (C) Time course of germination of WT, sense and antisense seeds in the water containing $15 \%$ PEG. The percentage of germinated seeds corresponds to the mean of three experiments of 50 seeds. (D) Seeds of WT (left), sense (middle) and antisense (right) lines corresponding to the $48 \mathrm{~h}$ time point in (C). Bar $=6 \mathrm{~mm}$ (B), Bar $=5 \mathrm{~mm}$ (D). 


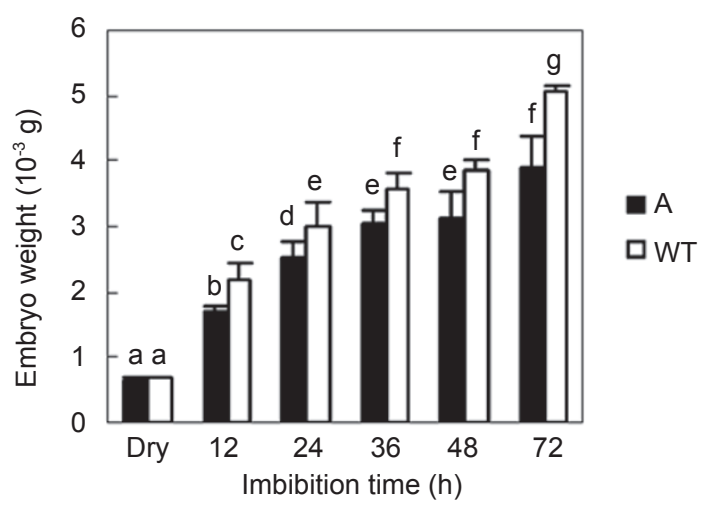

Figure 5 Comparison of the capacity of water uptake in the wild type and antisense $O S P I P 1 ; 3$ rice seed embryos at different stages of germination. At each time point, different letters above bars indicate significant differences at $P<0.05(0.01)$ as determined by one-way ANOVA followed by Turkey's test.

reduced in $O S P I P 1 ; 3$ antisense-transgenic seeds, we investigated the effects of gene silencing on water uptake during seed germination. We measured water uptake from detached wild type and antisense-transgenic seed embryos. Throughout the duration of imbibition, wild-type seed embryos consistently took up more water than antisensetransgenic seed embryos as determined by embryo weight $(P<0.05$ at 24 and $36 \mathrm{~h}, P<0.01$ at 12 and $48 \mathrm{~h}$ ) (Figure 5). These results indicate that the reduced seed germination rate in antisense-transgenic plants resulted from the decreased availability of water uptake. The sense-seeds and the wild-type seeds showed no difference when they were germinated under near optimal conditions, and there was no difference in their water uptake (data not shown).

\section{Function of NO on seed germination}

We investigated the effects of the exogenous NO donors, SNP and GSNO, and the NO scavenger PTIO on rice seed germination to examine the role of $\mathrm{NO}$ in this process. SNP releases NO when illuminated [29] or when it reacts with biological tissues [30]. Murgia et al. [31] reported that different NO donors could have different or even opposite effects on plant metabolism; therefore, we used two NO donors GSNO and SNP. An increase in the seed germination percentage was detected when rice seeds were germinated under light in the presence of $100 \mu \mathrm{M}$ SNP or $100 \mu \mathrm{M}$ GSNO for 24,30 or $36 \mathrm{~h}$ (Figure 6A). The results revealed that $\mathrm{NO}$ donors promote seed germination in the early stage of germination. Furthermore, the seed germination was delayed in $100 \mu \mathrm{M}$ PTIO (Figure 6A). In addition, when the NO scavenger PTIO was added to the solutions containing SNP or GSNO, the positive effects of SNP and
GSNO on seed germination were weakened (Figure 6A). It was reported recently that SNP, as well as potassium ferricyanide and potassium ferrocyanide, reduced dormancy of Arabidopsis seeds by generating cyanide [32]. To determine whether cyanide was the compound in SNP vapors that promoted rice seed germination, control experiments were carried out with potassium ferricyanide. There was no significant difference in the rate of germination and the final germination percentage between the seeds germinated in potassium ferricyanide and water (data not shown). These results suggest that exogenous NO can play a role in regulating rice seed germination. However, we could not rule out the possibility that SNP reduces dormancy of seeds by generating cyanide, because we did not directly investigate the effects of NO on seed dormancy in this study.

To document the effect of endogenous NO on seed
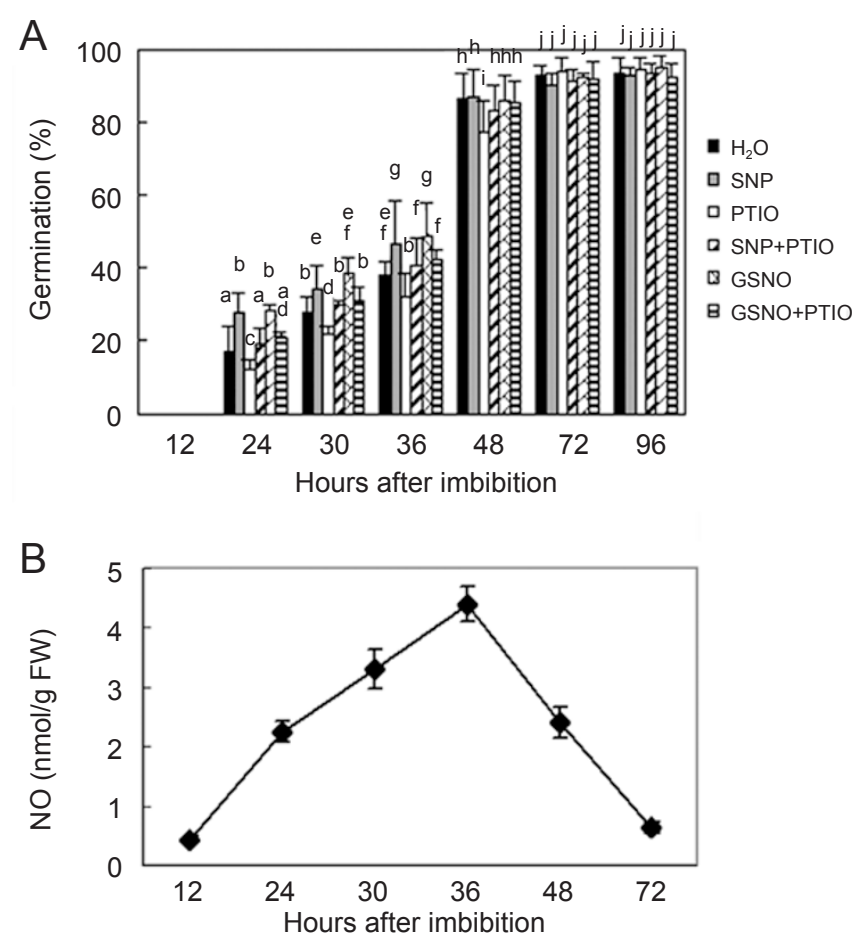

Figure 6 Effects of NO on seed germination. (A) The NO donors, SNP and GSNO, stimulated seed germination and the NO scavenger PTIO inhibited seed germination. Seeds were imbibed with distilled water alone, or imbibed with $100 \mu \mathrm{M}$ SNP, $100 \mu \mathrm{M}$ GSNO, 100 $\mu \mathrm{M}$ PTIO or $100 \mu \mathrm{M}$ SNP with $100 \mu \mathrm{M}$ PTIO, $100 \mu \mathrm{M}$ GSNO with $100 \mu \mathrm{M}$ PTIO. Values are the means \pm S.E.M. of three independent experiments. At each time point, different letters above bars indicate significant differences at $P<0.05$ as determined by one-way ANOVA followed by Turkey's test. (B) Nitrite concentration as an estimate for NO production in germinating seeds. Values are the means \pm S.E.M. of three independent experiments. 


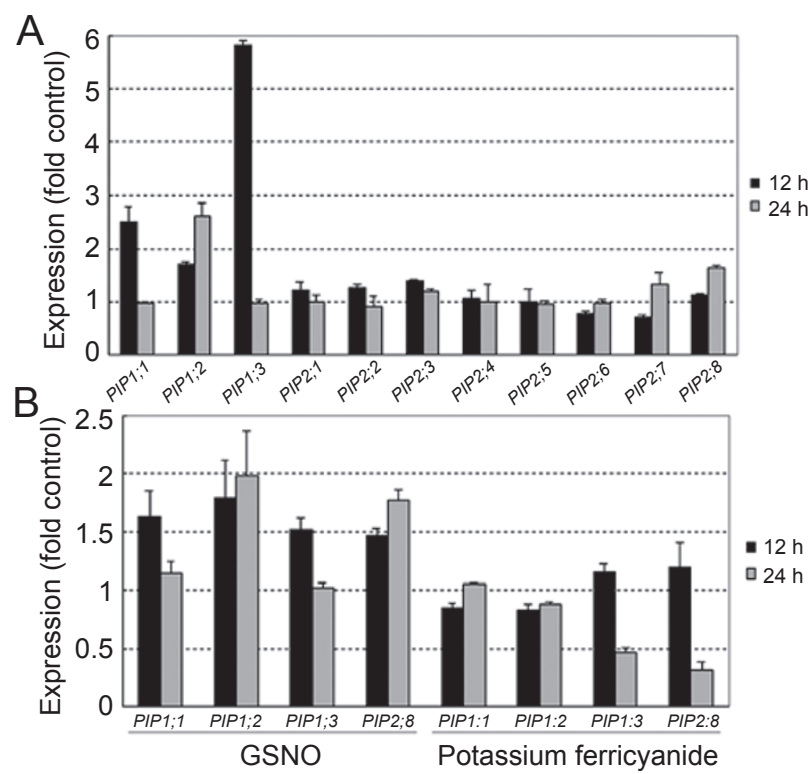

Figure 7 The response of $P I P$ expression to NO and cyanide. (A) SNP induced OsPIP 1;1, OsPIP1;2, OsPIP1;3 and OsPIP 2;8 expression in the embryos of germinating seeds. Seeds were imbibed with distilled water or $100 \mu \mathrm{M}$ SNP. (B) The effects of GSNO and potassium ferricyanide on $O s P I P 1 ; 1, O s P I P 1 ; 2, O_{S P I P 1 ; 3}$ and $O s P I P 2 ; 8$ expression. The relative expression of PIPs in the treated embryos was described as fold control (treated/control ratio). Values are the means \pm S.D. of three independent experiments.

germination, we imbibed seeds in water for $12,24,30$, 36,48 and $72 \mathrm{~h}$, and then analyzed NO concentration in embryos. As shown in Figure 6B, the content of NO in homogenates from embryos significantly increased between 12 and $36 \mathrm{~h}$ of imbibition, and sharply declined after $36 \mathrm{~h}$ of germination, coinciding with the initiation of an active germination.

Effects of NO on PIP transcript levels in germinating seed

Hormones such as $\mathrm{ABA}$ or $\mathrm{GA}_{3}$ and a variety of environmental stimuli regulate the transcription of water channel genes [33]. NO/cGMP signal transduction has a crucial role in $\mathrm{Ca}^{2+}$ homeostasis in the $\mathrm{M} 3$ muscarinic acetylcholine-stimulated increase in AQP5 levels in the apical plasma membrane of rat parotid glands [34]. NO plays a role in seed germination, yet little is understood about how it functions. The data presented here show that NO triggered certain PIP expression during seed germination. NO upregulated the expression of four of 11 rice PIPs (Figure 7A). As analyzed by real-time PCR, SNP caused a 2.6-fold increase in OSPIP1;1 transcript levels in germinating seeds after $12 \mathrm{~h}$ of treatment, and 1.7- and 2.6-fold increase in OsPIP1;2 transcript levels after 12 and $24 \mathrm{~h}$ of treatment, respectively (Figure 7A). SNP also increased OSPIP 1;3 expression by 5.8 -fold at $12 \mathrm{~h}$ after germination, but had no effect at $24 \mathrm{~h}$ after germination. GSNO also affected the expression levels of PIP1 mRNA in seeds. As shown in Figure 7B, the expression of OsPIP 1;1 was increased to 1.6 -fold at $12 \mathrm{~h}$ of imbibition. OsPIP $1 ; 2$ exhibited about 1.8- and 2.0-fold increase after 12 and $24 \mathrm{~h}$ of germination, meanwhile, OsPIP 1;3 expression at $12 \mathrm{~h}$ after germination was increased to 1.5 -fold in treated embryos. Among the eight members of the rice PIP2 sub-family, only OSPIP $2 ; 8$ was significantly induced by the application of exogenous NO. 1.6- and 1.8-fold increase in OSPIP 2;8 transcript levels were detected in germinating embryos after treatment with SNP and GSNO for $24 \mathrm{~h}$ compared with the control. Treatment with SNP did not result in an increase in transcript levels of any PIPs after $36 \mathrm{~h}$ of germination (data not shown). In order to determine if the effect of SNP on PIP expression is the result of NO or the result of cyanide, it is necessary to know the response of the PIP to cyanide. The response of PIP expression to the treatment of potassium ferricyanide solution is shown in Figure 7B. These data show that potassium ferricyanide at a concentration of $100 \mu \mathrm{M}$ did not result in enhancement of OsPIP1 and OsPIP 2;8 expression; on the contrary, it reduced their expression at certain time points. Although the NO donors could promote germination of seeds from OsPIP1;3 antisense lines, the final germination percentage of these seeds was still lower than that of the wild-type seeds (data not shown). On the basis of the above data, we conclude that the effects of $\mathrm{NO}$ on rice seed germination may be partly mediated through induction of water channels and therefore enhanced ability of water uptake.

\section{Discussion}

The presence of PIP1 members in dry and germinating seeds has been noted before. The mRNA of PSPIP1;1 or its possible homologs was detected in the dry and germinating seeds, suggesting that they could play a role in water absorption during seed imbibition [35]. It was also shown previously that the water channel proteins, BnPIP1and BnTIP2, play a role in seed germination [9]. The authors speculated that BnPIP1 was involved in water transport required for the activation of enzymatic metabolism of storage nutrients in the early stages of canola seed germination, while BnTIP2 expression was correlated with cell growth during radicle emergence. To gain insight into the role of water channel family in germination, we investigated the expression of PIP1 and PIP2 subfamilies in rice seed. The time course of PIP expression in embryos 
showed that it was developmentally regulated. The expression of OsPIP 1;1, OsPIP1;2, OsPIP1;3, OsPIP 2;1, OsPIP 2;4, OsPIP 2;5 and OsPIP2;8 increased strongly in the embryo a few hours before radicle emergence. This expression profile indicated that they might be involved in the processes linked to seed germination. Other PIPs could also play an important role in the movement of water during seed germination, because OSPIP 2;2, OsPIP 2;6 and OsPIP 2; 7 existed in germinating seeds. In the embryos of dry seeds, except for $O_{S P I P 1 ; 3, O S P I P 2 ; 4}$ and $O s P I P 2 ; 5$, other $O s P I P$ s were present at different expression levels. It is noteworthy that $O S P I P 2 ; 3$ was not expressed until radicle emergence, followed by increased expression with seedling growth. These results suggest that $O S P I P 2 ; 3$ does not contribute to seed germination but functions in plant growth. Both the PIP1 and PIP2 subfamilies might also be involved in the developmental process of the early phases of post-germination, because all were still present after radicle emergence.

Previous studies have revealed that water channels exist in almost all types of tissues [2, 28], and their crucial functions have been demonstrated in many plants $[33,36]$. They comprise a relatively large gene family. For example, there are 35 water channel homologs in Arabidopsis [5] and 33 members in maize [6]. At least 33 water channel genes have been identified in the rice genome [3]. A series of papers have elucidated the function and regulation of PIP. These water channels are likely to play different roles in different cells and expected to be regulated differently. In the present experiment, we tried to clarify the function of water channel in seed germination by over-expressing and silencing $O S$ PIP 1;3. Our results indicated that the sense-transgenic rice seeds germinated faster than wild-type seeds upon waterdeficit treatment. Yu et al. [37] have also demonstrated that over-expression of BnPIP1 in transgenic tobacco resulted in earlier seed germination upon drought condition than the untransformed control. Water channel proteins appear to be functionally sufficient when seeds germinate under normal conditions, since wild-type seeds have the ability to take up enough water for germination. Silencing water channel expression seems to reduce the ability of seeds to absorb water as a result of the water channel deficiency. However, PIP1 isoforms have often been found to have very low, or no, water permeability when expressed solely in Xenopus oocytes [22, 38-40]. Previously, Chaumont and his co-workers [41] have demonstrated that interactions between maize ZmPIP1;1 and ZmPIP1;2 or between ZmPIP1;2 and ZmPIP2 positively modulated their water channel activity. Hence, PIP1-members may function as water channels in seed germination via PIP interactions and these interactions enhance their water channel activity. There is another possibility that PIP1-members are not directly involved in water transport, but are related to other physiological functions in seed germination.

Previous reports have demonstrated that water channels are regulated by many factors including developmental and environmental factors [42]. The transmembrane water flux via the regulation of aquaporin quantity and activity endows plants with a remarkable capacity to modulate water absorption, transport and compartmentation within tissues. In animal studies, the role of NO/cGMP signal transduction in the M3 muscarinic acetylcholine receptor-stimulated increase in AQP5 levels in the apical plasma membrane of rat parotid glands was reported [34]. Pretreatment of rat parotid tissue with the NO scavenger inhibited acetylcholine-induced increases in AQP5 in the apical plasma membrane. NO donors mimicked the effects of muscarinic acetylcholine receptor agonists. However, the possible role of NO signalling in changes of PIP levels in plants was unknown. In this study, we revealed the involvement of NO in regulation of PIP expression as observed in the response of germinating seeds to exogenous NO treatment. The phylogenetically divergent subgroups of water channels may reflect isoforms with different biochemical properties and may fulfill unique or diverse functions in plant development, and hence their patterns of regulation are expected to differ. Here, the three PIP1 genes, OsPIP1;1, OsPIP1;2 and OsPIP1;3, expressed in germinating rice seeds, were classified by phylogenetic analysis as belonging to the same subgroup [3]. They also exhibited similar developmental patterns of expression and were regulated similarly by exogenous NO. Most other members of the PIP 2 group did not respond to exogenous NO except OsPIP 2;8; and it has a unique sequence different from the others in the family. This suggests that the regulation of individual water channels may be tied to their physiological functions in each organ.

Together, the data presented here indicate that the PIPs play an important role in seed germination. The reduced seed germination rate via $O S P I P 1 ; 3$ silencing and the promotion of seed germination via $O S P I P 1 ; 3$ over-expression under drought conditions demonstrated clearly that OsPIP1;3, as well as perhaps OsPIP1;1, is required for normal seed germination. In addition, our data also suggest that $\mathrm{NO}$ can promote rice seed germination and certain water channel proteins could function in this process as downstream effectors of the NO signaling pathway. Specific rice NO signaling mutants and other PIP transgenic plants are necessary to better understand NO-mediated signal transduction and its relationship with water channel proteins in plant growth and development.

\section{Acknowledgments}

We thank Dr David Lane for correction of the English in 
the manuscript. This work was supported by the National Natural Science Foundation of China (No. 30670172) and by the Korea Foundation for International Cooperation of Science and Technology (KICOS) through a grant provided by the Korean Ministry of Science and Technology.

\section{References}

1 Bewley JD. Seed germination and dormancy. Plant Cell 1997; 9:1055-1066.

2 Maurel C. Aquaporins and water permeability of plant membranes. Annu Rev Plant Physiol Plant Mol Biol 1997; 48:399429.

3 Sakurai J, Ishikawa F, Yamaguchi T, Uemura M, Maeshima M. Identification of 33 rice aquaporin genes and analysis of their expression and function. Plant Cell Physiol 2005; 46:1568-1577.

4 Yu X, Peng YH, Zhang MH, Shao YJ, Su WA, Tang ZC. Water relations and an expression analysis of plasma membrane intrinsic proteins in sensitive and tolerant rice during chilling and recovery. Cell Res 2006; 16:599-608.

5 Johanson U, Karlsson M, Johansson I. The complete set of genes encoding major intrinsic proteins in Arabidopsis provides a framework for a new nomenclature for major intrinsic proteins in plants. Plant Physiol 2001; 126:1358-1369.

6 Chaumont F, Barrieu F, Wojcik E, Chrispeels MJ, Jung R. Aquaporins constitute a large and highly divergent protein family in maize. Plant Physiol 2001; 125:1206-1215.

7 Kaldenhoff R, Fischer M. Functional aquaporin diversity in plants. Biochim Biophys Acta 2006; 1758:1134-1141.

8 Bouton S, Viau L, Lelievre E, Limami AM. A gene encoding a protein with a proline-rich domain (Mtpprd1), revealed by suppressive subtractive hybridization ( $\mathrm{SSH}$ ), is specifically expressed in the Medicago truncatula embryo axis during germination. J Exp Bot 2005; 413:825-832.

9 Gao YP, Young L, Bonham-Smith P, Gusta LV. Characterization and expression of plasma and tonoplast membrane aquaporins in primed seed of Brassica napus during germination under stress conditions. Plant Mol Biol 1999; 40:635-644.

10 Beligni MV, Lamattina L. Nitric oxide stimulates seed germination and de-etiolation, and inhibits hypocotyl elongation, three light-inducible responses in plants. Planta 2000; 210:215-221.

11 Finkelstein RR, Lynch TJ. Abscisic acid inhibition of radicle emergence but not seedlings growth is suppressed by sugars. Plant Physiol 2000; 122:1179-1186.

12 Neill SJ, Desikan R, Hancock JT. Nitric oxide signaling in plants. New Phytol 2003; 159:11-35.

13 Crawford NM, Guo FQ. New insights into nitric oxide metabolism and regulatory functions. Trends Plant Sci 2005; 10:195200.

14 Delledonne M. NO news is good news for plants. Curr Opin Plant Biol 2005; 8:390-396.

15 Sakihama Y, Nakamura S, Yamasaki H. Nitric oxide production mediated by nitrate reductase in the green alga Chlamydomonas reinhardtii: an alternative NO production pathway in photosynthetic organisms. Plant Cell Physiol 2002; 43:290-297.

16 Batak I, Devic M, Giba Z, Grubisic D, Poff KL, Konjevic R. The effects of potassium nitrate and NO-donors on phytochrome Aand phytochrome B-specific induced germination of Arabidopsis thaliana seeds. Seed Sci Res 2002; 12:253-259.

17 Bethke PC, Gubler F, Jacobsen JV, Jones RL. Dormancy of Arabidopsis seeds and barley grains can be broken by nitric oxide. Planta 2004; 219:847-855.

18 Bethke PC, Libourel IGL, Jones RL. Sodium nitroprusside, cyanide, nitrite, and nitrate break Arabidopsis seed dormancy in a nitric oxide-dependent manner. Planta 2005; 223:805-812.

19 Kopyra M, GwóŸdŸ EA. Nitric oxide stimulates seed germination and counteracts the inhibitory effects of heavy metals and salinity on root growth of Lupinus luteus. Plant Physiol Biochem 2003; 41:1011-1017

20 Simontacchi M, Jasid S, Puntarulo S. Nitric oxide generation during early germination of sorghum seeds. Plant Sci 2004; 167:839-847.

21 Kim KY, Kwon SY, Lee HS, Hur Y, Bang JW, Kwak SS. A novel oxidative stress-inducible peroxidase promoter from sweetpotato: molecular cloning and characterization in transgenic tobacco plants and cultured cells. Plant Mol Biol 2003; 51:831-838.

22 Lian HL, Yu X, Ye Q, et al. Water channel protein RWC3 is involved in the drought avoidance in rice. Plant Cell Physiol 2004; 45:481-489.

23 Liu QQ, Zhang JL, Wang ZY, Hong MM, Gu MH. A highly efficient transformation system mediated by Agrobacterium tumefaciens in rice (Oryza sativa L.). Acta Phytophysiol Sinica 1998; 24:259-271 (in Chinese).

24 Nishi R, Hashimoto H, Kidou S, Uchimiya H, Kato A. Isolation and characterization of a rice cDNA which encodes a ubiquitin protein and a 52 amino acid extension protein. Plant Mol Biol 1993; 22:159-161.

25 Ohshima Y, Iwasaki I, Suga S, Murakami M, Inoue K, Maeshima M. Low aquaporin content and low osmotic water permeability of the plasma and vacuolar membranes of a CAM plant Graptopetalum paraguayense: comparison with radish. Plant Cell Physiol 2001; 42:1119-1129.

26 Bradford MM. A rapid and sensitive for the quantitation of microgram quantities of protein utilizing the principle of protein-dye binding. Anal Biochem 1976; 72:248-254.

27 Sambrook F, Fritsch EF, Maniatis T. Molecular cloning: a laboratory manual. 2nd Edition. New York: Cold Spring Harbor Laboratory Press, 1989:1860-1865.

28 Sun MH, Zhang MH, Liu HY, et al. Distribution of water channel protein RWC3 and its regulation by GA and sucrose in rice (Oryza sativa). Acta Bot Sin 2004; 46:1056-1064.

29 Feelisch M. The use of nitric oxide donors in pharmacological studies. Naunyn Schmiedebergs Arch Pharmacol 1998; 358:113122.

30 Bethke PC, Badger MR, Jones RL. Apoplastic synthesis of nitric oxide by plant tissues. Plant Cell 2004; 16:332-341.

31 Murgia I, de Pinto MC, Delledonne M, Soave C, De Gara L. Comparative effects of various nitric oxide donors on ferritin regulation, programmed cell death, and cell redox state in plant cells. J Plant Physiol 2004; 161:777-783.

32 Bethke PC, Libourel IGL, Jones RL. Nitric oxide reduces seed dormancy in Arabidopsis. J Exp Bot 2006; 57:517-526.

33 Luu DT, Maurel C. Aquaporins in a challenging environment: molecular gears for adjusting plant water status. Plant Cell Environ 2005; 28:85-96.

34 Ishikawa $Y$, Iida $\mathrm{H}$, Ishida $\mathrm{H}$. The muscarinic acetylcholine receptor-stimulated increase in aquaporin-5 levels in the apical plasma 
membrane in rat parotid acinar cells is coupled with activation of nitric oxide/cGMP signal transduction. Mol Pharmacol 2002; 61:1423-1434.

35 Schuurmans JAMJ, van Dongen JT, Rutjens BPW, Boonman A, Pieterse CMJ, Borstlap AC. Members of the aquaporin family in the developing pea seed coat include representatives of the PIP, TIP, and NIP subfamilies. Plant Mol Biol 2003; 53:655-667.

36 Hachez C, Zelazny E, Chaumont F. Modulating the expression of aquaporin genes in planta: a key to understand their physiological functions? Biochim Biophys Acta 2006; 1758:1142-1156.

$37 \mathrm{Yu}$ Q, Hu Y, Li J, Wu Q, Lin Z. Sense and antisense expression of plasma membrane aquaporin BnPIP1 from Brassica napus in tobacco and its effects on plant drought resistance. Plant Sci 2005; 169:647-656.

38 Kammerloher W, Fischer U, Piechottka GP, Schaffner AR. Water channels in the plant plasma membrane cloned by immunoselection from a mammalian expression system. Plant J 1994; 6:187199.

39 Chaumont F, Barrieu F, Jung R, Chrispeels MJ. Plasma membrane intrinsic proteins from maize cluster in two sequence subgroups with differential aquaporin activity. Plant Physiol 2000; 122:1025-1034

40 Moshelion M, Becker D, Biela A, et al. Plasma membrane aquaporins in the motor cells of Samanea saman: diurnal and circadian regulation. Plant Cell 2002; 14:727-739.

41 Fetter K, Van Wilder V, Moshelion M, Chaumont F. Interactions between plasma membrane aquaporins modulate their water channel activity. Plant Cell 2004; 16:215-228.

42 Maurel C, Javot H, Lauvergeat V, et al. Molecular physiology of aquaporins in plants. Int Rev Cytol 2002; 215:105-148. 